\title{
Experimental and Kinetic Modeling Studies of 3-Methylfuran Pyrolysis at Low and Atmospheric Pressures
}

Huaijiang Su${ }^{1}$, Jinglan Wang 2 , Jiabiao Zou 3 , Qiang Xu ${ }^{1}$, Jiuzhong Yang 4 , Zhanjun Cheng ${ }^{2, *}$, Lixia $\mathrm{Wei}^{1, *}$

${ }^{1}$ College of Mechanical Engineering, Guangxi University, Nanning, Guangxi, 530004, P. R. China

${ }^{2}$ School of Environmental Science and Engineering, Tianjin University, Tianjin, 300072, P. R. China

${ }^{3}$ Key Laboratory for Power Machinery and Engineering of MOE, Shanghai Jiao Tong University, Shanghai, 200240, PR China

${ }^{4}$ National Synchrotron Radiation Laboratory, University of Science and Technology of China, Hefei, Anhui, 230029, P. R. China 
Table S1 Name and structure of selected species in the model

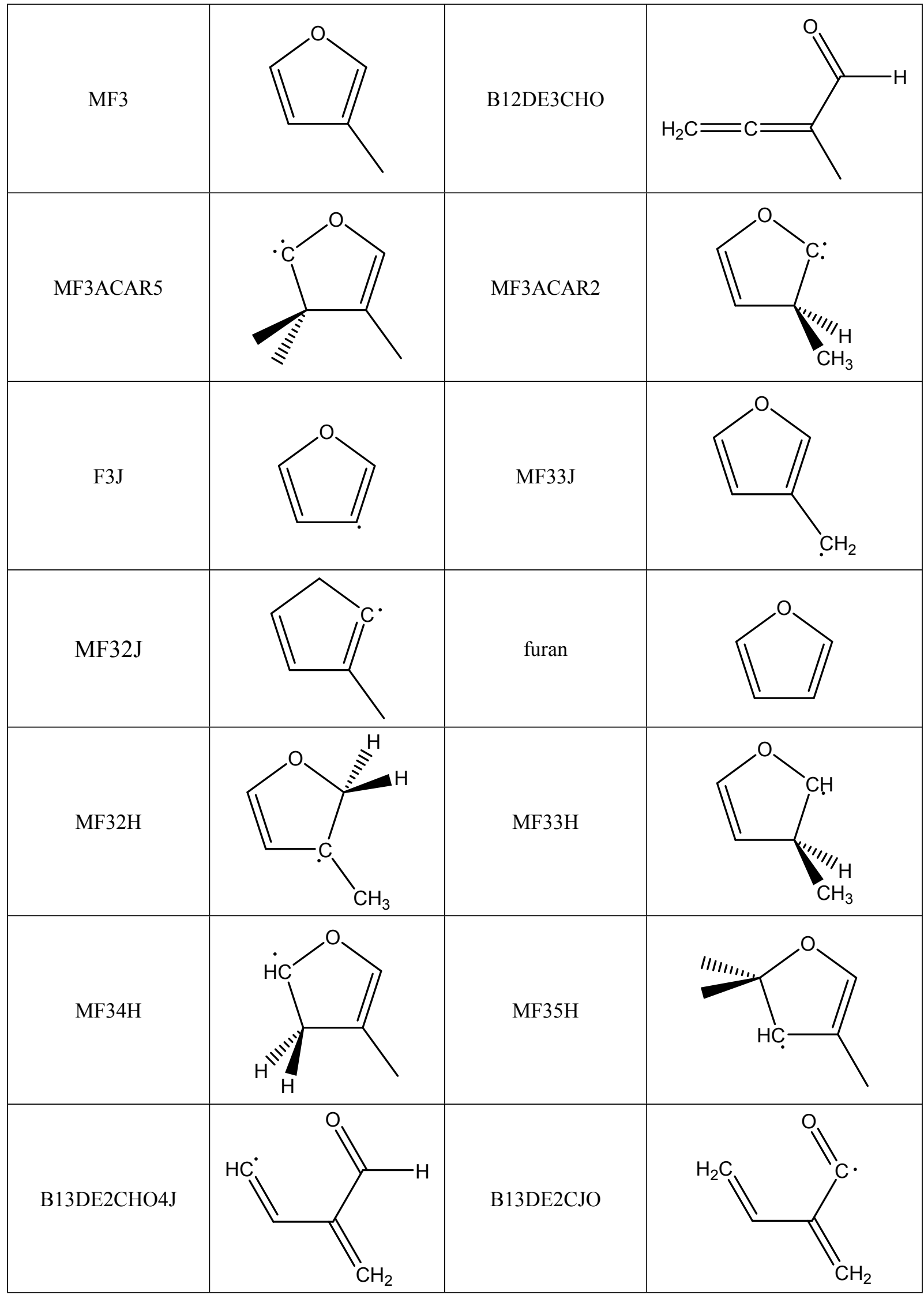



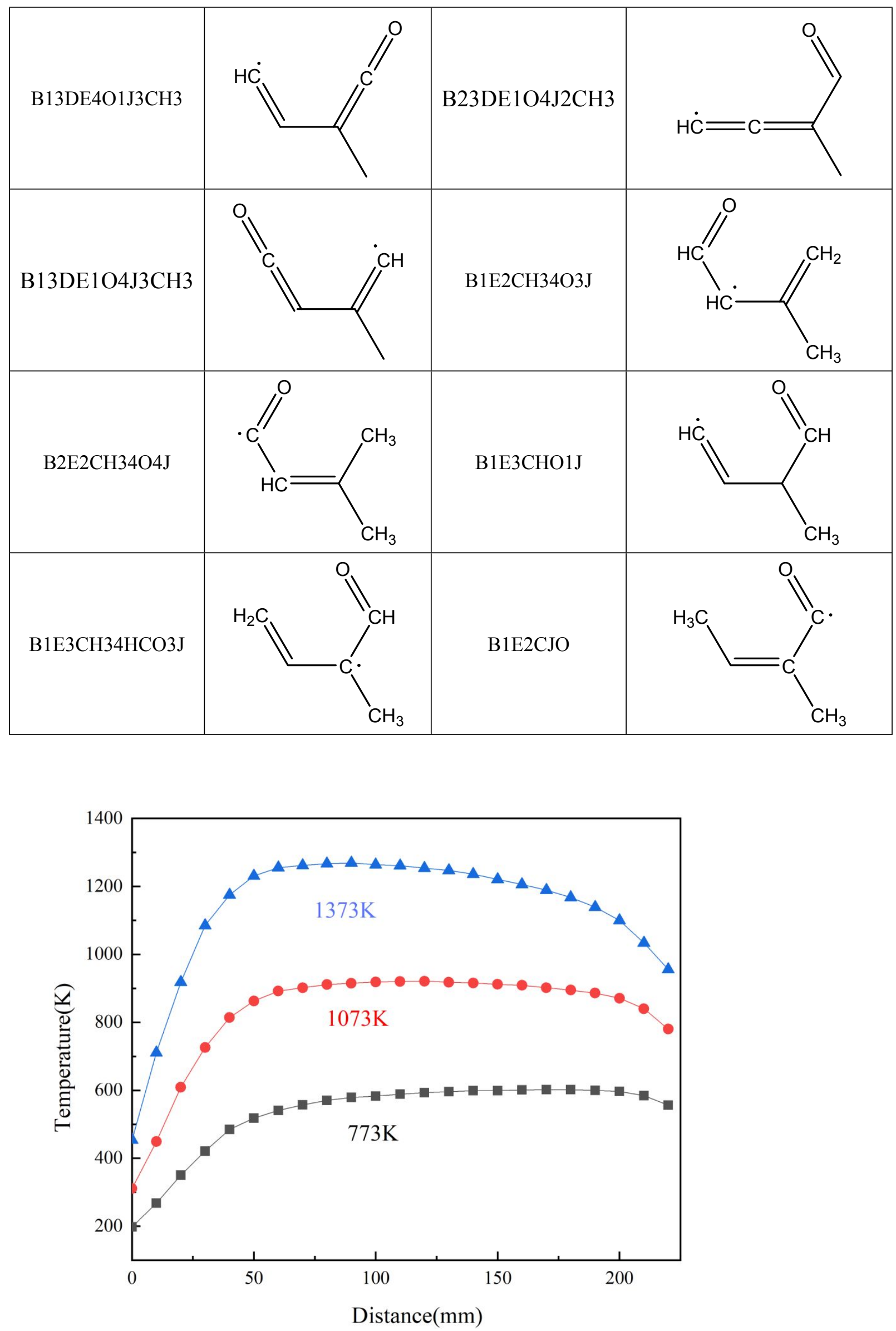
Figure S1 Axial temperature profiles of the flow tube measured in this work. 